\title{
Exacerbation of Alphaviral Arthritis and Myositis in a Mouse Model after Etanercept Treatment is due to Diminished Levels of Interferon $\alpha / \beta$
}

\section{Ali Zaid ${ }^{1}$, Kuo-Ching Sheng ${ }^{1}$, Adam Taylor ${ }^{1}$, Nestor E Rulli', Lara J Herrero' ${ }^{1}$, Patrick McNeil H $^{2}$ and Suresh Mahalingam ${ }^{1 \star}$}

${ }^{1}$ Emerging Viruses and Inflammation Research Group, Institute for Glycomics, Griffith University, Gold Coast, Australia

${ }^{2}$ South Western Sydney Clinical School, University of New South Wales, Sydney, Australia

Little is known about the possible use of TNF- $\alpha$ inhibitors for treating viral arthritis and myositis caused by mosquitoborne arthritogenic alphaviruses such as Ross River virus (RRV), chikungunya virus (CHIKV), and Barmah Forest virus. Emerging alphaviruses such as CHIKV, Mayaro virus and O'nyong-nyong virus are now considered significant public health threats by the World Health Organisation (WHO), with reports of severe cases of debilitating arthritis and arthralgia due to these agents in numerous regions of the world [1]. Although alphavirus-induced arthritis is generally self-limiting, the disease can be severe and the virus can persist and establish chronic infection [1]. For example, Hourau et al. recently showed the persistence of CHIKV antigen in perivascular synovial macrophages in a patient 18 months after infection [2]. At present, there are no alphavirus vaccines or antiviral drugs available for humans. The current treatments available for symptoms caused by alphavirus disease consist of non-steroidal anti-inflammatory drugs such as aspirin or ibuprofen [1].

In recent years we have identified key mechanisms of viral arthritis using our mouse model of alphavirus-induced arthritides and have been investigating ways to reduce joint and muscle inflammation. In this model, macrophages are prominent within the synovial joints and high levels of pro-inflammatory cytokines such as TNF- $\alpha$ and MCP-1 are produced [3]. This is similar to the synovial joints of RRV patients suffering from polyarthritis, in which these soluble factors and macrophages are also prominent. These observations suggest that a considerable immunopathological component is involved in alphavirus-induced arthritis [3]. Targeting specific inflammatory mediators within the joint provides a possible therapeutic approach to alleviate the disease. Indeed, depletion of macrophages by administration of clodronate in mice resulted in marked improvement of the disease. Similarly, the pharmaceutical compound bindarit, an indazole derivative capable of inhibiting MCP-1 production and monocyte recruitment, has a beneficial therapeutic effect on alphavirus disease in mice $[4,5]$. In our recent work [6], we determined whether inhibiting TNF- $\alpha$ in vivo could ameliorate the alphaviral arthritis, based on the therapeutic efficacy of this treatment in autoimmune diseases. Surprisingly, we found that treating alphavirus-infected mice with etanercept exacerbated muscle and joint inflammation, with increased cellular infiltration in the synovium and muscle fibres [6]. In addition, viral titres were higher in etanercept treated mice, and this associated with high morbidity [6]. These results indicated that TNF- $\alpha$ has a protective role during alphavirus infection in mice and highlights the important role of this cytokine in antiviral immune responses. The differential effects of anti-TNF- $\alpha$ treatment in autoimmune inflammation and viral arthritis reflects the pleiotropic nature of this cytokine: while TNF- $\alpha$ is pro-inflammatory by inducing cell activation, apoptosis and tissue destruction, it is also a critical activator of signalling pathways, such as NFKB and MAPK, that are indispensible for production of various cytokines and chemokines to achieve immune competency [7]. To this end, TNF- $\alpha$ has been linked to the action of a critical class of cytokines, type I interferons (IFNs) [7]. TNF- $\alpha$ can stimulate IFN- $\beta$ production and initiate an autocrine loop that sustains IFN-induced gene expression [7]. Furthermore, TNF- $\alpha$ synergises with IFN- $\beta$ to enhance chemokine production and the antiviral response [7]. Type I IFNs potently inhibit alphavirus replication and have been proposed as a potential treatment. Based on the interrelationship between TNF- $\alpha$ and type I IFNs, we hypothesized that the exacerbation of alphaviral arthritis by etanercept was due to alterations in the level of type I IFNs. Indeed, IFN- $\alpha$ and IFN- $\beta$ levels were greatly reduced or undetectable in mice infected with RRV and treated with etanercept (Figure 1A and 1B). These results suggest that anti- TNF- $\alpha$ dampens host anti-viral immunity by inhibiting the production of type
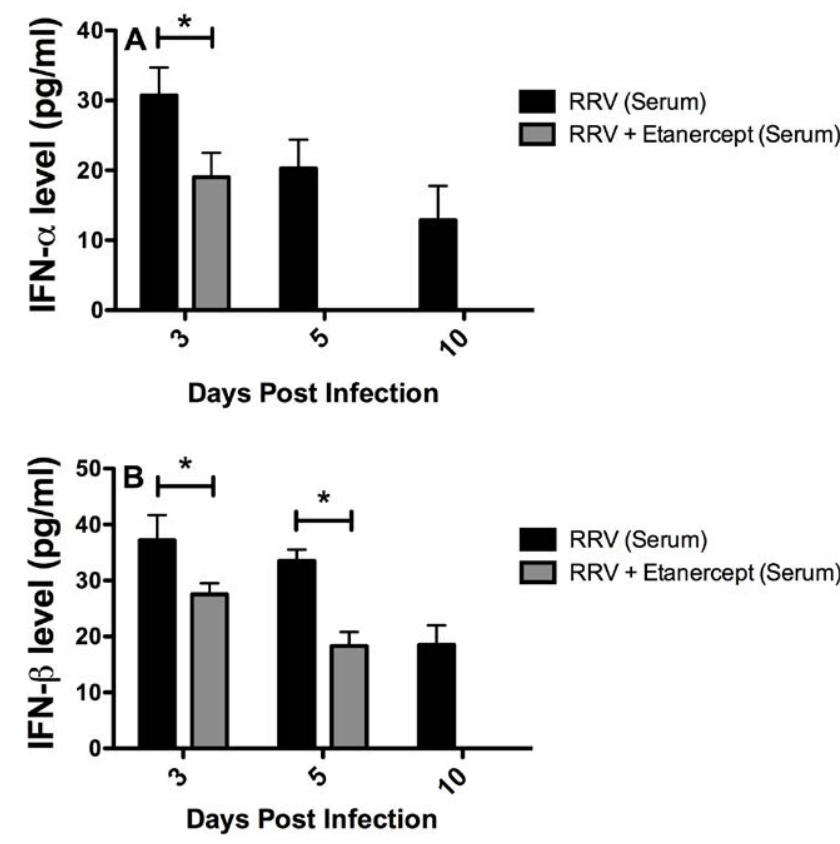

Figure 1: Type I IFN levels in serum of RRV-infected C57BL/6J mice treated with etanercept. Mice were infected subcutaneously with 103 pfu of RRV. Mice received peritoneal injections of etanercept on days $1,3,5$ and 7 post-infection. IFN- $\alpha(A)$ and IFN- $\beta$ (B) levels in the serum of RRV and RRV-etanercept mice at 3,5 and 10 days post-infection. Significant differences are marked by an asterisk. *, $\mathrm{P}<0.05$ (for untreated infected versus etanercept treated infected; $\mathrm{n}=5$ ). This study was approved by the University of Canberra Animal Ethics Committee (Protocols: CEAE 06-14 -and CEAE 08-14).

${ }^{*}$ Corresponding author: Suresh Mahalingam, Emerging Viruses and Inflammation Research Group, Institute for Glycomics, Griffith University (Gold Coast Campus), Queensland 4222, Australia, Tel: +61 75552 7178; Fax: +61 75552 8098; E-mail: s.mahalingam@griffith.edu.au

Received October 17, 2013; Accepted October 19, 2013; Published October 22 2013

Citation: Zaid A, Sheng KC, Taylor A, Rulli NE, Herrero LJ, et al. (2013) Exacerbation of Alphaviral Arthritis and Myositis in a Mouse Model after Etanercept Treatment is due to Diminished Levels of Interferon $\alpha / \beta$. Virol Mycol 2: 122. doi:10.4172/2161-0517.1000122

Copyright: () 2013 Zaid A, et al. This is an open-access article distributed under the terms of the Creative Commons Attribution License, which permits unrestricted use, distribution, and reproduction in any medium, provided the original author and source are credited. 
Citation: Zaid A, Sheng KC, Taylor A, Rulli NE, Herrero LJ, et al. (2013) Exacerbation of Alphaviral Arthritis and Myositis in a Mouse Model after Etanercept Treatment is due to Diminished Levels of Interferon $\alpha / \beta$. Virol Mycol 2: 122. doi:10.4172/2161-0517.1000122

I IFN. The potency of etanercept in this model is, therefore, likely due to dual inhibition of the synergistic partners TNF- $\alpha$ and type I IFN.

Side effects of etanercept were officially reported by the US health authorities, following the death of some etanercept treated patients as a result of severe sepsis [8]. Etanercept treatment has also been associated with severe viral respiratory tract infections in humans [9]. In addition, patients receiving anti-TNF- $\alpha$ therapy may be associated with an increased risk of viral infections like human immunodeficiency virus, varicella zoster virus, Epstein-Barr virus, cytomegalovirus and pappilomavirus $[9,10]$. Reactivation of latent infection with $M$. tuberculosis has been reported following anti-TNF- $\alpha$ treatment, and patients contemplating anti-TNF- $\alpha$ therapy should be screened for latent infection before therapy [9]. We suggest that RA patients receiving anti-TNF- $\alpha$ drugs would be more susceptible to alphavirus infection, and clinicians must be particularly aware of the possibility of viral infection as a cause of disease exacerbation in RA patients receiving anti-TNF- $\alpha$ drugs, particularly in areas where arthrogenic alphaviruses are endemic. Anti-TNF- $\alpha$ therapy should be avoided for the clinical treatment of alphavirus-induced arthritis as it may dampen the anti-viral activity of type I IFNs that are essential for viral clearance. In conclusion, patients presenting with arthritic symptoms should be screened for alphavirus serum antibodies as a precaution before being prescribed anti-TNF- $\alpha$ therapy for RA.

\section{Acknowledgment}

This study was supported by a grant from the Australian National Health and Medical Research Council (NHMRC Project Grant 508600).

\section{References}

1. Burt FJ, Rolph MS, Rulli NE, Mahalingam S, Heise MT (2012) Chikungunya: a re-emerging virus. Lancet 18: 662-671.

2. Hoarau JJ, Jaffar Bandjee MC, Krejbich TP, Das T, Li-Pat-Yuen G, et al. (2010) Persistent chronic inflammation and infection by Chikungunya arthritogenic alphavirus in spite of a robust host immune response. J Immunol 184: 59145927.

3. Lidbury BA, Rulli NE, Suhrbier A, Smith PN, McColl SR, et al. (2008) Macrophage-derived proinflammatory factors contribute to the development of arthritis and myositis after infection with an arthrogenic alphavirus. J Infect Dis 197: 1585-1593.

4. Rulli NE, Guglielmotti A, Mangano G, Rolph MS, Apicella C, et al. (2009) Amelioration of alphavirus-induced arthritis and myositis in a mouse model by treatment with Bindarit, an inhibitor of monocyte chemotactic proteins. Arthritis Rheum 60: 2513-2523.

5. Rulli NE, Rolph MS, Srikiatkhachorn A (2011) MCPs inhibitor ameliorates Chikungunya disease in a mouse model of arthritis and myositis. J Infect Dis 204: 1026-1030.

6. Zaid A, Rulli NE, Rolph MS, Suhrbier A, Mahalingam S (2011) Disease exacerbation by Etanercept in a mouse model of alphaviral arthritis and myositis. Arthitis Rheum 63: 488-491.

7. Yarilina A, Ivashkiv LB (2010) Type I interferon: a new player in TNF signaling Curr Dir Autoimmun 11: 94-104.

8. Nash PT, Florin THJ (2005) Tumour necrosis factor inhibitors. Med J Aust 183 205-208.

9. Winthrop KL (2006) Risk and prevention of tuberculosis and other serious opportunistic infections associated with the inhibition of tumor necrosis factor. Nat Clin Pract Rheumatol 2: 602-610.

10. Kim SY, Solomon DH (2010) Tumor necrosis factor blockade and the risk of viral infection. Nat Rev Rheumatol 6: 165-174. 